\title{
Quantitative electron ptychography for simultaneous light and heavy elements atom counting
}

\author{
E. Liberti ${ }^{1,2}$, A. N. Moya ${ }^{1}$, K. P. Treder ${ }^{1}$, C. M. O’Leary ${ }^{1}$, J. S. Kim ${ }^{1,2,3}$, P. D. Nellist ${ }^{1}$, A. I. Kirkland ${ }^{1,2,3}$ \\ ${ }^{1}$ Department of Materials, University of Oxford, Oxford, UK, ${ }^{2}$ electron Physical Science Imaging Centre (ePSIC), Diamond Light \\ Source, Didcot, UK, ${ }^{3}$ The Rosalind Franklin Institute, Harwell Campus, Didcot, UK
}

emanuela.liberti@materials.ox.ac.uk

In scanning transmission electron microscopy (STEM), real space image quantification allows the counting of the number of atoms in a crystallographic projection, perpendicular to the electron probe. Atom counting is an established method in high-angle annular darkfield (HAADF) imaging and has applications that include the estimation of 3D shapes in metallic nanoparticles [1-2], or local composition variations in high-Z materials with known, constant thickness [3]. The incoherent nature of the HAADF contrast yields a monotonic increase of the image counts with $Z$, and this allows us to directly interpret image contrast at atomic columns positions as the number of atoms in projection. However, HAADF is only valid for atom counting of heavy elements that scatter strongly at highangle. Phase imaging techniques are more appropriate for counting light elements because they ensure the collection of weak scattering signals at low angles. Phase methods recover the phase of the electron wavefunction scattered by both light and heavy elements; however, this phase is not directly quantifiable due to coherence [4]. Here, we present a novel approach to phase quantification, based on the combination of HAADF atom counting and electron ptychography. Electron ptychography is a 4D STEM phase technique whereby we recover the complex exit wavefunction from a set of $2 \mathrm{D}$ coherent electron diffraction patterns collected over a 2D image grid. Unlike other phase recovery techniques, electron ptychography has the unique advantage that it can be performed simultaneously with HAADF imaging. This allows for atom counting of heavy elements which can be used as means to rescale the ptychographic phase and count the light elements [5-6]. Herein, we apply this novel quantitative ptychographic approach to determine the local sub-stoichiometric composition of $\mathrm{CeO}_{2-\mathrm{x}}$ nanoparticles. Fig. 1 (a) illustrates a schematic of the 4D STEM ptychographic method, where the Ce atom count in (b), obtained from the simultaneously recorded HAADF image, is used to calibrate the ptychographic phase in (c) to count $\mathrm{O}$ atoms.

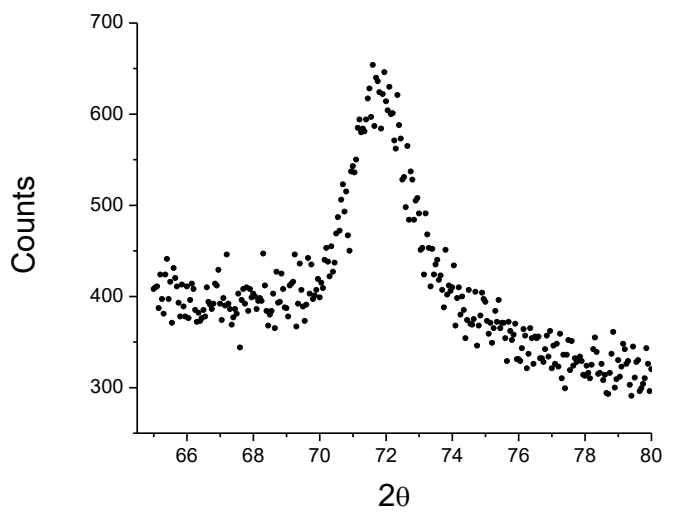

Figure 1. Schematic of quantitative electron ptychography applied to the measurement of local stoichiometry in ceria.

[1] L. Jones et al., Nanolett. 14 (2014) 6336.

[2] S. Van Aert et al, Phys. Rev. B 87 (2013) 064107.

[3] A. Rosenauer et al., Ultramic. 109 (2009) 1171.

[4] D. Van Dyck et al., Nature 486 (2012) 243.

[5] H. Yang et al., Nat. Comm. 7 (2016) 12532.

[6] A. De Backer et al., Ultramic. 171 (2016) 104.

Keywords: electron ptychography; image quantification; atom counting. 